\title{
Role of Machine Vision for Identification of Kidney Stones Using Multi- Features Analysis
}

\author{
Hafiz Muhammad Farjad ${ }^{1}$, Salman Qadri², Mubashir Hussain Malik ${ }^{3}$, Najia Saher ${ }^{4}$, Faisal Shahzad ${ }^{5}$, Syed Ali \\ Nawaz $^{6}$ and Dost Muhammad Khan ${ }^{7}$ \\ ${ }^{1,3}$ Department of Computer Science, Institute of Southern Punjab Multan \\ ${ }^{2}$ Department of Computer Science MNS-University of Agriculture Multan \\ ${ }^{4,5,6,7}$ Department of Information Technology, The Islamia University of Bahawalpur \\ Email: Salman.qadri@mnsuam.edu.pk
}

(Received: 15 March 2021 ; Accepted: 12 April 2021; Issue Published: 12 September 2021)

\begin{abstract}
The purpose of this study is to highlight the significance of machine vision for the Classification of kidney stones identification. A novel optimized fused texture features framework was designed to identify the stones in the kidney. Each region of interest (ROI) acquired a fused 234 texture feature (GLCM, RLM and Histogram) feature set was acquired by each region of interest (ROI). It was observed that on each image 8 ROI's of sizes (16x16, $20 \times 20$ and $22 \times 22)$ were taken. It was difficult to handle a large feature space 280800 (1200x234). Now to overcome this data handling issue we have applied a feature optimization technique namely POE+ACC and acquired 30 most optimized features set for each ROI. The optimized fused features data set 3600(1200x30) was used to four machine vision Classifiers that is Random Forest, MLP, j48 and Nä̈ve Bayes. Finally, it was observed that Random Forest provides best results of 90\% accuracy on ROI $22 x 22$ among the above discussed deployed Classifiers.
\end{abstract}

KEYWORDS: Region of Interest (ROI), Grey Level Co-occurrence Matrix (GLCM), Run Length Matrix (RLM), Multi-Layer Perception (MLP)

\section{INTRODUCTION}

Kidney is the major organ of the human body. It is used for purification of blood and it separates the poisonous material from human body. Healthy kidney filters about a half cup of blood every minute to separate the poisonous material from human body through urine. There are different types of diseases of kidney. One of the most common diseases is stones in kidney. Diagnose the stone in the kidney, it's very difficult in the early stage because there are no symptoms shown in human body. It is an excruciating disease in human body. In medical science, different techniques are used to identify the stone in kidney (Xray, ultra-sonic MRI and CT scan). It is impossible to realize the problem at early stage of disease, due to non-specific symptoms and it can be easily checked only when the disease starts damaging the organ. Xray is used to detect the size and position of stones in kidney [1]. Furthermore, kidney disease, which damages the kidneys, leads to be undone and poses problem.

Consequently, it is important to detect kidney stone disease before the disease does damage permanently. If the stone problem is caught in the early stage, the disease very effectively can be treated. Consequently, the diagnosis of stone is important not only for the treatment of kidney disease but also for managing the persistent formation of stone. A few years ago, 3D medical image processing played a very important part in computer-aided diagnosis, which also supports radiologists in assessing in recognizing abnormal findings in medical imagery. Traditional tests like a biopsy, urine test and blood test and imaging test. MRI, CT and ultrasound are utilized to diagnose kidney stone disease. According to the deliberation, which is based on cost, time-taken and information increased on imaging tests, diagnostic tests using computer tomography (CT) have developed theost common one between diagnostic tests in [2]. To detect chronic kidney disease (CKD), neural networks have been used and other pathologies associated to the urinary system. Some models have made it possible to detect kidney stones [3] [4]. Uric acid, creatinine, lymphocytes, glucose and other blood components from the results that are taken in the laboratory. Other work has focused on predicting the survival of patients with CKD [5].

Over the last few decades, Kidney Stone(KS) blocking the urine flow in the upper urinary tract, is due to stones in the kidney [6]. In the starting stage, kidney stone infections stay unnoticed, which affect the kidney as they are found. 
The leading cause of kidney failure is glomerulonephritis, hypertension, diabetes mellitus, etc. in the starting stages, kidney disease is advisable. The UltraSound Result is one of the most available strategies [7].

\section{LITERATURE REVIEW}

In the medical field, to diagnose the different diseases for kidneys by using (CT, X-ray and MRI) for the Picture creating of live tissues. Since the circumstances are favorable for ultrasound imaging strategy, for example, convenience of the device, being fewer expensive, security of the imaging process to the patient and the less amount of real-time prerequisite for imaging. It has been given more understanding than other imaging techniquessed for kidney diseases [8].

The main loss of kidney function has been Chronic Renal Disease (CRD)for a few decades. At the early stage of detection, CKD is crucial and very helpful in declining medical resources as ESRD patientsreserve their health through peritoneal dialysis and kidney transplantation [9]. By using Creatinine (CR) and the blood urea nitrogen (BUN), index is used in early detection diagnosis for (CKD) which is usually acquired through blood testing. The patients can be analyzed using ultra sound images as a methodological solution to diagnose efficiently [10].

The ultra-sonic imaging technique is less effective for patients who may predict that ratio is low because the tissue and fat get deeper, so its intelligibility becomes lower in Ultrasound rely, which basically depends on the skills of the technician for accuracy. The results of CT scan images give a very higher contrast image to identify the interior size, structure, density, design and kidney. On CT scan images technique, kidney stone diagnosing is still a challenge in division because it is too complex structures of interest in abdomen CT. In dissimilar nature of the tissues, resemblances among the contiguous organs, lack of clear boundaries, partial-volume effect, noise, accurate segmentation of the organs are very difficult issues. Kidney stone is the most hazardous problem worldwide. It is essential to analyze the kidney stone at an early stage by human expert (radiologist/nephrologist) to provide effective treatment. Many imaging techniques like ultra-sound, X-ray, CT scans and MRI images are used to diagnose kidney stones. Radiologists take the history of the patient and if they find the symptoms of kidney stone, they use imaging techniques, mostly CT scans to verify the exact location of the stone. Radiologists have to scan the cross-section images generated by the CT (Computed tomography) scan images, mark the stone area, and label them manually. To do this work, human experts must have the full knowledge of kidney anatomy otherwise, wrong marking may provide wrong results and this can cause very serious problem. Moreover, these scanning and marking procedures are very tedious and time consuming. So, I try to find out the method / system that assists the radiologist in diagnosing the kidney stone as soon as possible. Many technologies like image processing, Artificial Intelligence and data mining are used to resolve the difficulty in earlier kidney stone diagnosis. These technologies help the physician and radiologist for the detection of kidney stone and early diagnosis stones in kidney. Computerized Tomography images (CT) scan has been identified as accurate non-invasive imaging technology in the diagnosis of kidney stones and it has several advantages over other technologies. Experienced doctors and radiologists use these techniques to detect the kidney stone location because the process after the location of stone is difficult, timeconsuming and based on human vision and may create some issue to diagnose. That's why it has been tried to identify the stone through machine vision. Manual diagnosis procedure, which expert radiologist does, requires proper attention and full kidney anatomy knowledge. To remove such limits, a semi-automatic diagnosis system is introduced to support radiologist who uses the output from a computerized analysis of medical images in lesions detection and makes diagnostic decisions, proposes a model, estimates and diagnoses the chronic kidney diseases by using Machine learning. Support Vector Machine (SVM) is more suitable for CKD results in kidney patients and high rate results were obtained from it. Support Vector Machine and decision tree algorithm are used for early detection of CKD. This is very important for treatment cost classification and the health of the patient. Two different types of datasets are used for the training and testing process of algorithms. Different types of predictions algorithms are performed on different sizes of data. This process is very effective and is widely used for the estimation of diseases [11].

A model was developed by using ultrasound in deep learning for classification and automation of the kidney function prediction. Ultrasound imaging has been considered as safe, convenient and affordable for the prediction of CKD. They develop the estimation of GFR in CKD. The creatinine-based GFR is estimated by artificial intelligence (AI). This model was considered a real time distinct screening tool and is an effect fundamental steps to Wald realizing the potential of transforming kidney [12].

Model for computer-aided detection (CAD) of ureteral stones in thin slice using convolutional neural networks $(\mathrm{CNN})$ was presented. Ureteral stones are identified in thin slice computer tomography (CT) by using computer aided detection algorithm. They work 
CNN directly on the high resolution (CT) volumes. The best model was $2.5 \mathrm{D}$ for achieving $100 \%$ sensitivity. The main problem was the extremely small size of stone found in disturbed ureter. The study of small size stones differentiates from the previous work in several ways, because they use raw pixels on 3D volumes, instead of extracting features using anatomical information. Texture features are used on the non-contrast CT images are used in this technique. For Computer aided detection most, recent work is used in renal stone for the workflow of noise detection and MSER features [13].

A system is suggested for the prediction and diagnosing of chronic kidney (CKD). The proposed model also measures the performance of our VMs model. "There are two techniques used in their system: one is Neural Network (NN) and second is linear regression (LR)". Linear Regression is used for determining the critical section and (NN) is used in diagnose for the (CKD) to improve the result for efficiency regarding real-time execution and improving the accuracy [14].

"In this study, 10 years clinical data is used for different variables of the newly diagnosed CKD patients". A model was developed for predicting chronic kidney diseases by using Integrated Intelligent Fuzzy Expert System and predicted renal failure progression their model is used to find the value of GFR and has achieved the results Normalized MAE that is lower than $5 \%$. This model accurately predicts the GFR variation, Despite the uncertainties of dynamic nature of CKD progression and human body [15].

A model was developed that uses three different types of supervised learning algorithm (multi-Layer perception, Genetic Algorithm and Decision tree algorithm). For the prediction of risk of kidney stones, Multi-layer perception is used as the best predictive model [16].

It proposed a model to classify and analyze different mineral components presented in kidney stones using microscopic Raman spectroscopy. This system was used to observe four different types of kidney stones (oxalates, phosphates, purines and L-cystine). Two different types of machine learning approaches are used to classify the results (PCA-KNN, PCA-SVM). The system gives results diagnostic accuracies $96.3 \%$ for the PCA-KNN and PCA-SVM methods with high sensitivity $(0.963,0.963)$ and specificity $(0.995$, 0.985) [17]. A model was developed to identify risk of CKD using Neural Network (NN) machine learning approaches (ML) with five Layers that are hidden and support vector machine (SVM). This model trains two types of data, one is diagnosed with $\mathrm{CKD}$, and the second is without a diagnosis. The accuracy of $95 \%$ is achieved in the dataset that is tested in this method. For the explanation of CKD predictions, they apply twin system and validate a Neural Network (NN). About 3,494,516 people were identified as being at risk of developing CKD as the result of this system [18].

There was Offered a system for the classification of Kidney stones. For capturing images of expelled kidney stones, they design a new device and give a new method of an expert system for classification. Eight classes of Kidney stones taxonomy are used in this system that give 63\% accuracy [19].

Put forward a system to predict the early stages Chronic Kidney Disease (CKD) in kidney using different machine learning techniques such as Logistic Regression, Naive Bayes, Artificial Neural Networks and Decision Trees. The proposed system depends on different steps, which are as follows: data extraction, acquire data into database, input attribute values, predict the presence of CKD using Naive Bayes, extract the attribute required for stage, calculate Glomerular Filtration Rate (GFR) and predict stagebased value for Glomerular Filtration Rate (GFR). This System provides a decision support tool that can help in the diagnosis of CKD [20].

The proposed model uses the eleven-layer of specific a three-dimensional Convolutional Neural Network (3DCNN) eleven layers deep for lesion segmentation. Dual pathway architecture is used in this model that processes the input images in multiple scales such as, Input segmentation (High grade and Low Grade), CNN (layers) Brain MRI, and Classification Layer. The proposed model evaluates three different tasks about brain tumors, lesion segmentation in multichannel MRI patient data and ischemic stroke with traumatic brain injuries. A more discriminative and deeper network with low cost and less number of parameters, a new model is developed [21].

It was proposed that a diagnostic system is to identify the difference between kidney stones and phlebitis. The accuracy of radionics is investigated in this system, that also checks the machine learning classifier in that is used to differentiate between phlebitis on LDCT and kidney stones. The proposed method consists of number of phases which are Image acquisition, Imaging analysis and reference standard, Phlebolith and Kidney stones segmentation on LDCT, Radionics features extraction and Machine-learning Classification model. The overall accuracy of $85.1 \%$ is achieved in this system on the independent testing set [22].

It was proposed a diagnostic model named- kidney stones (DACA-KS) by using laboratory data and demographic and integrating large volume of data". The Proposed model were utilized to look at 
affectability, particularity and region under the beneficiary working trademark (AUROC) of our methodology with the STONE score, utilizing crossapproval. The framework gives results in multi-space approach utilizing calculated relapse yielded an AUROC of 0.86 and an affectability/explicitness of $0.81 / 0.82$ in cross-approval. Increment in execution was acquired by fitting a super-student, at the cost of lower interpretability [23].

Advanced a semi-automated model Kidney-UrineBelly computed tomography (KUB CT) for enhancing kidney stone segmentation and detection of kidney area using geometry principles and image processing technique. The Proposed system depends on different steps as follows: Localization (Bounding Box), Contrast Adjustment (Gamma Adjustment), Segmentation (Threshold), Combining the Connected Component Labeling, Slices Using Logical OR Operation, Removing the Ribs and Spine Restriction and Object Detection, Virtual Region Segmentation, Design Restriction to Segmented Region and Output. The proposed model gives results with an accuracy of $84.61 \%$ [24].

Submitted a system that detects lung cancer. Detection of lung cancer is based on fuzzy logic and it classifies the normal and abnormal images using a neural network. The Proposed system depends on different phases such as Feature CT image, pre-processing, Enhancement, Segmentation, Features Extraction, Neural network Classifier (normal or abnormal) and Diagnosis Result. The system produced good results in several images [25]. A system was proposed to diagnosed CKD in the early stages using SVM algorithm. The system gives the results $93 \%$ of experimental data sets based on three performance metrics, i.e., sensitivity, specificity and accuracy [26]. A system was suggested for the prediction of chronic kidney diseases using new decision support systems. Advocated system has many phases that are used of CKD, Data Mining classification (KNN and SVM and Performance Evaluation. K-NN gives the better accuracy performance as compared to SVM, Pre and gives the less execution time for the prediction of CKD [27].

Recommended a system for chronic kidney diseases using Multiple feature selection methods independently. The system combines all the results to find the optimal solution. The system generates the best performance on the random forest Classifiers [28]. A system increasing the accuracy of CKD diagnosing was proposed. This system uses selected features with the property of Particle Swarm Optimization (PSO) features. This system reduces redundancies and produces optimal features. This system also used ensemble AdaBoost to increase the performance of entirety Classification algorithm [29]. Initiated system for Kidney disease detection using artificial neural network. This system depends on different phases such as ultrasound image as an input image. it is preprocessing, feature extraction, feature selection, Classification using ANN, normal images and abnormal images, segmentation. It uses multi kernel $\mathrm{K}$ means clustering in the abnormal region. The presented system achieves the maximum accuracy of $99.61 \%$ by using linear and quadratic based segmentation [30].

A system for finding the transitional interval of the kidney at 3-5 different stages by using data techniques Like ANN, Decision tree, K-Nearest Neighbor for the eliciting knowledge and creating a Classification model with the selected attributes was proposed. This system is used as a decision support tool [31]. Presents two effective supervised learning methods to improve the exactness of the kidney stones Classification. The proposed procedure picture incorporates apparently abused by urologists to perceive the sort of kidney stones are destitute down and encoded as vectors. Classification is performed on these part vectors through Random Forest and assembling K Nearest Neighbor Classifiers. The overall Classification precision procured was $89 \%$ [32]. A deep learning method by using digital images for the identification of kidney stones was proposed. Multi-Class Classification Model is used in deep CNN to each image. The overall weighted REC of the CNNs composition analysis was $85 \%$ [33].

They have named a model to diagnose chronic diseases by using the Support Vector Machine Classification algorithm. To diagnose CKD, two basic sorts of highlight choice techniques specifically, covering and channel approaches, were picked to lessen the Chronic Kidney Disease dataset component. The outcomes demonstrated the Support Vector Machine Classifier by utilizing separated sub set evaluator with the Best First web index include choice strategy has higher precision rate $(98.5 \%)$ in the analysis of chronic kidney disease [34]. Put-up a model for identification of the large kidney stone. It uses Statistical machine learning methods and Multivariate logistic regression to infer prediction models". This proposed model provides better results to identify the kidney stone [35].

Proposed a model to detect and diagnose chronic kidney disease using Hetero Generous Modified Artificial Neural Network (HMANN). A model was developed that includes supervised machine learning techniques and dimensionality reduction (Fishers) and Classification of large-sized kidney stone identification. The derived DSS was assessed by running forget about one-quiet cross-approval 
approach on the dataset. The framework gave good exactness (94.8\%) [36]. The following are as different steps in the proposed model: image input, image preprocessing, feature extraction, image segmentation, wavelet processing, ANN Classification, and identifying the stone. The model significantly reduces the time and also gives the high accuracy [37].

\section{PROPOSED METHODLOGEY}

The proposed methodology of kidney stone identification frame work (KSIF). There are different steps given as follows.

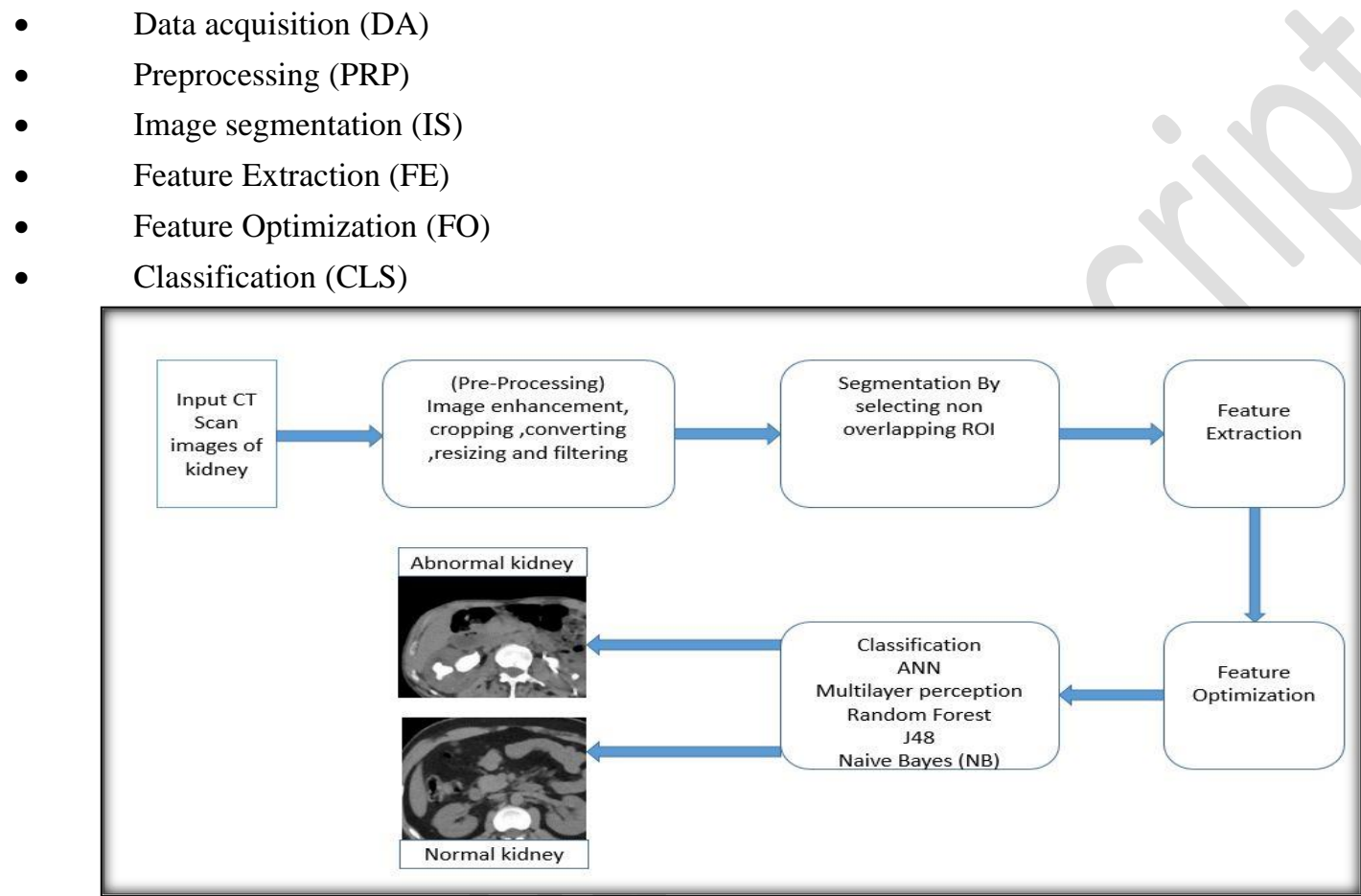

Figure 1: Proposed methodology

\subsection{Data Acquisition}

A data set of 50 patients CT scan images with Toshiba Aquiline 64 slice CT scanner and obtained from "Bahawal Victoria Hospital Bahawalpur", Pakistan was acquired for experiment evaluation. It consists of 150 kidney CT scan images out of 75 CT scan images are normal and $75 \mathrm{CT}$ scan images are abnormal kidney. The kidney CT scan images in DICOM format first, we convert the images in jpeg format. Figure 3.2 and 3.3 show a sample of normal and abnormal CT images respectively selected for experimentation. First, we have $150 \mathrm{CT}$ images of the kidney in the resized form $(256 \times 256)$. The second, step is converted into the 8-bit gray-scale and stored in Bitmap (BMP) format. Figure 3.4 and 3.5 show the grayscale image result. Eight non-overlapping Region of interest (ROI's) at different pixels dimension were acquired to extract the maximum information from the CT images. Figure 4.6 shows a sample of kidney eight non-overlapping (ROI).
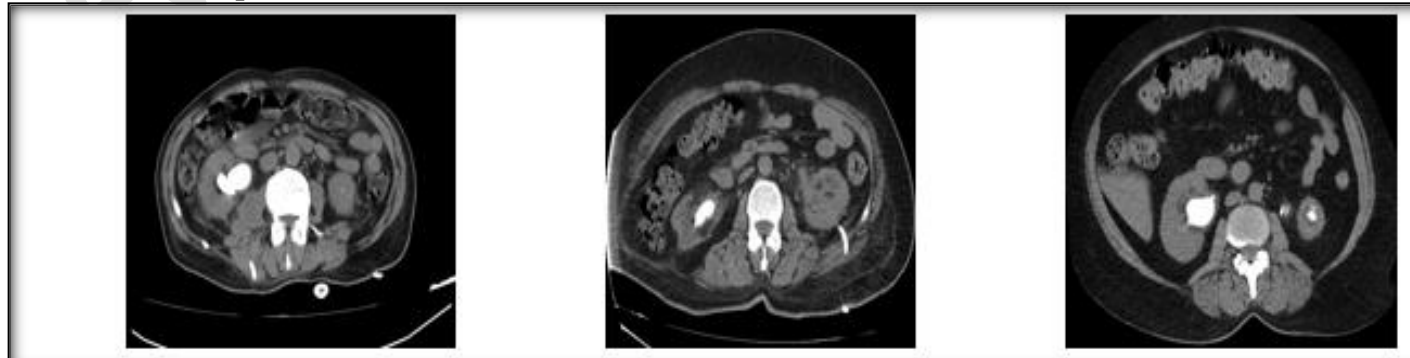

Figure 2: CT scan images for abnormal kidney 


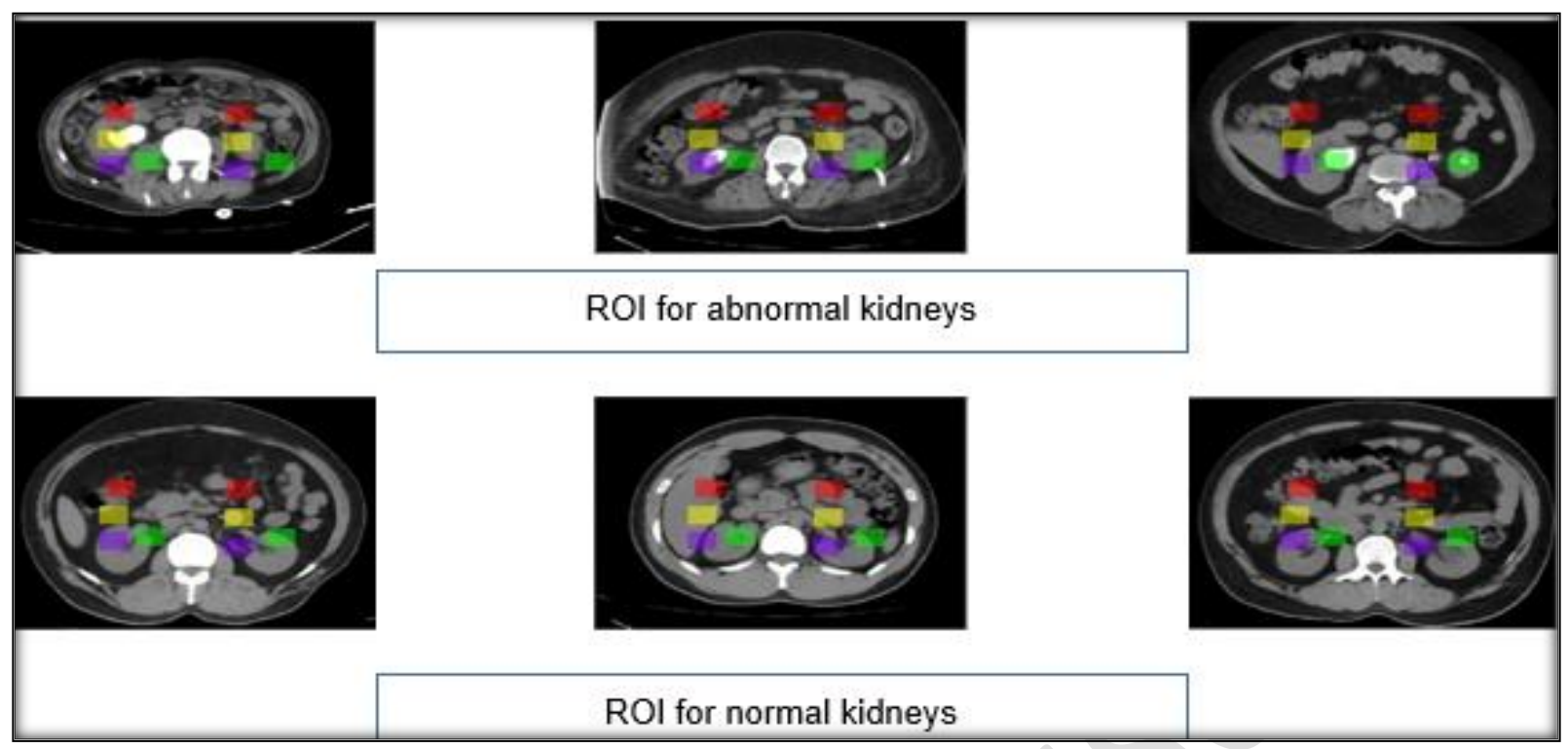

Figure 3: Gray scale kidney images of eight non-overlapping (ROI)

\subsection{Preprocessing}

It is the important task in the detection of abnormalities in kidney. Frequently the medical images appeared inhomogeneous and poor contrast that requires preprocessing that enhances the quality of images. These steps are following in preprocessing . (Resize the image, convert into gray level representation, apply filter and Cropping the image).

\subsection{Image segmentation}

Segmentation is the preliminary and significant step in image analysis to extract information from complex medical images. Image segmentation used to separate the actual part of kidney from the undesired. It is the process where the image is partitioned into multiple segments so that processed images is easier to analyze.

\subsection{Feature Extraction}

In feature extraction phase to extract the different three types of features are extracted from all kidney CT scan images dataset. First one is histogram, second is Run length Matrix (RLM) and third one is Co-occurrence Matrix. These features are including nine histograms , 5 run length matrix and 11 co-occurrence matrix three different sizes of ROIs $(16 \times 16,20 \times 20,22 \times 22)$ in all four $\left(0^{0}, 45^{0}, 90^{0}, 135^{\circ}\right)$ dimensions. It was developed large feature space 280800 (1200x234). All features were extracted using Mazda.

\subsubsection{First Order histogram}

We are used to first order histogram to extract the features from the image dataset of kidney CT scan. The histogram shows the pixels intensity.

The method commonly used for image feature extraction is based upon the first-order histogram.
Pixel intensity is used to calculate histogram without considering any structural relationships between image and pixels. Characteristics are simply statistical parameters of the histogram distribution, such as variance, kurtosis, mean brightness, skewness and percentiles. The average describes the values in average form and these show the average value in the dark and light tones.

\subsubsection{Run-length matrix (RLM)}

Run-length Matrix (RLM) contains the number of runs of pixels that have a given grayscale level and length. Mazda calculates four matrices of different series lengths for the four-pixel run directions (00 450900 and 1350). There are different types of functions extracted using RLM, all images show below inverse moments of short-term emphasis, moments of longterm emphasis, gray level without uniformity, runlength unevenness, image fraction in series, and coefficient $\mathrm{C}$.

\subsubsection{Co-occurrence matrix (COM)}

It is a second-order histogram, in which pairs of pixels are compiled from intensities, where the structural relationship of pair of pixels is defined. Texture features are calculated with the help of co-occurrence matrices, and each of them shows specific image characteristics such as contrast, coarseness, homo Genuity, and texture difficulty. Most commonly used are ("Angular second moment, Sum of squares, Sum average, Sum entropy, Entropy, Contrast, Correlation, Difference variance, Sum variance, Inverse difference moment and Difference entropy").

\subsection{Feature Optimization}

Features selection is an important role of research, with hundreds to thousands of space object datasets 
exist. It purposes the methodology that is used to specify more important features of the applied techniques. Moreover, reliable classification results are based on a large number of features; usually, big data is required, which is not easy to obtain. It is necessary to reduce the dimension of the vector space of statistical features, which allows one to distinguish and classify different types of these Classes of kidney. These approaches were used to select the most discriminant feature set. We applied these three data approaches: Fisher's coefficient, mutual information, and probability of error $(\mathrm{POE})+$ mean correlation (ACC) to reduce the feature vector space [38]. (POE) + (ACC) gives more accurate results compared to Fisher's coefficient and mutual information using Mazda. To show (POE + ACC) in equation number (i).

$$
F=\frac{D}{V}=\frac{\frac{1}{1-\sum_{k=1}^{K} P_{k}^{2}} \sum_{k=1}^{K} \sum_{j=1}^{K} P_{k} P_{j}\left(\mu_{k}-\mu_{j}\right)^{2}}{\sum_{k=1}^{K} P_{k} V_{k}}
$$

Mathematical relation for Mutual Information (MI) Coefficient is explained in equation (ii).

$$
\begin{aligned}
& M I\left(f_{j}, d\right)= \\
& \sum_{d=1}^{N b} \sum_{k=1}^{N c} P\left(f_{j}^{d}, C_{k}\right) \log _{2}\left[\frac{P\left(j_{j}^{d}, C_{k}\right)}{P\left(f_{j}^{d}\right) P\left(C_{k}\right)}\right]
\end{aligned}
$$

Fisher Coefficient (F) mathematically is described in equation number (iii).

$$
F=\frac{D}{V}=\frac{\frac{1}{1-\sum_{k=1}^{K} P_{k}^{2}} \sum_{k=1}^{K} \sum_{j=1}^{K} P_{k} P_{j}\left(\mu_{k}-\mu_{j}\right)^{2}}{\sum_{k=1}^{K} P_{k} V_{k}}
$$

\subsection{Classification}

Classification is a process where a given sample is assigned as a class on the basis of knowledge gained by classifier during training. Extracted features are given as input to classify the objects in an image. The two basic types of classifier are used in machine learning approach first one is supervised learning approach and the second one is unsupervised learning approach are as follow:

In this work, different data classification methods are used for kidney dataset. We have applied various classification procedures using WEKA software version (3.9) such as Multilayer Perceptron (MLP), Naïve Bayes (NB), Random Forest (RF) and J48. These Classifier's are used for two types of datasets such as texture and spectral. After applying feature selection and reduction techniques, all Classifiers are implemented to obtain the best overall accuracy results. Both types of datasets are organized for processing in Weka software into the Attribute Relation File Format (ARFF).

\section{RESULTS AND DISCUSSIONS}

In this chapter, we have discussed experiments and results for proposed methodologies implemented by using Mazda. The image processing tool version 4.6 with machine learning software WEKA version 3.9 on core I5 processor $1.95 \mathrm{GHz}$ with 8 gigabytes random access memory. A total of 2 different categories of kidney images (KI) are acquired 75 images of each. So, the total size of the image dataset is $(75 \times 2=150)$ which is collected for experimentation. These images convert into (.BMP) format and $256 \times 256$ size. After segmentation process, images are open in Mazda tool for image enhancement 8 region of interest ROI are selected on each image for texture analysis. Three types of features are extracted namely histogram, Runlength matrix and co-occurrence matrix feature. Analysis is carried the all features individually which are most appropriate for kidney stone texture analysis. After that all features are fused and the multi-features dataset is generated, 150 kidney stone and 8 ROI on each of the instances or size of the extracted dataset is 280800 (1200x234=280800). When data is fused, more attributes are combined; so, it is necessary to reduce or optimize the texture feature vector space. The feature selection method Probability of error (POE), Average correlation Co-efficient (ACC) is implemented, which selects the 30 optimized features were acquired dataset $3600(1200 \times 30=3600)$. These selected features are analyzed in the B11 tool, which is integrated with the Mazda tool. For classification using machine learning Classifier's namely Multilayer Perceptron (MLP), Naïve Bayes (NB), Random Forest (RF) and J48 with cross-validation 12-fold approach. After these experimentations, a comparative analysis of which features which classifier gives more accuracy in less time.

\subsection{Selected Optimize Features}

Table 1 shows the POE+ACC selected 30 features for experimentation; further analysis performs on this optimized dataset. 
Table 1. Optimized Features using POE+ACC

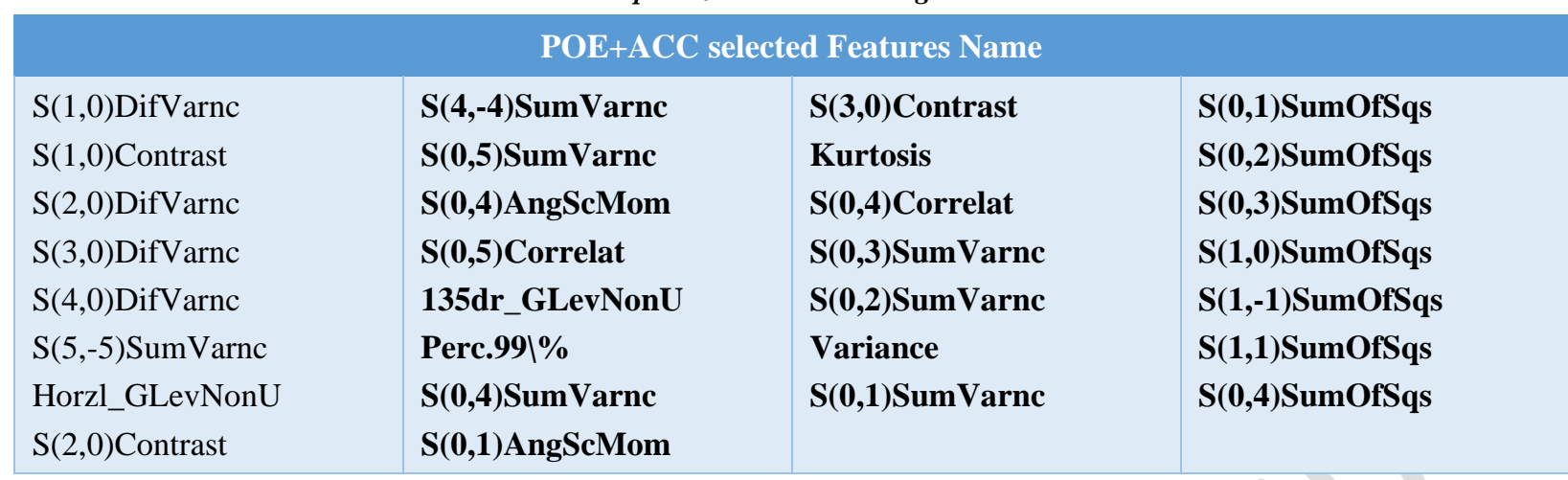

Performance calculating parameters learned classification accuracy results are as given below .

True PR:

It is known as True PR and is calculated as

True $P R=$ True $P R /($ True $P R+$ False NR)

True NR:

It is known as True NR and is calculated as

True NR = True NR /(True NR + False PR $)$

False PR:

It is known as False PR and is calculated as

False PR = False PR /(False PR + True NR)

False NR:

It is known as False NR and is calculated as

False NR = 1- True PR

Pre: Prec $=$ True PR $/($ True $\mathbf{P R}+$ False PR $)$

Rec $=$ True PR /(True PR + False NR)

F-Measure:
F-MEAS $=2 *$ Prec $*$ Rec $/($ Pre + Rec $)$

ROC A:

Graphical representation of classifiers between True PR and False PR.

CLASS:

There are two different classes used in this research normal and abnormal.

Confusion matrix:

It shows a diagonal matrix Classified as. These parameters show the performance of the implement Classifier.

Different tables are given below to show the different ROI sizes on different results using four machine learning classifiers (Random Forest, MLP, j48 and naïve Bayes).

Table 2. Classifiers Summary with ROI size (16x16)

\begin{tabular}{|c|c|c|c|c|c|c|c|}
\hline Classifiers & CCI & InCCI & KS & MAE & RMSE & RAE & RRSE \\
\hline MLP & $82 \%$ & $18 \%$ & 0.64 & 0.2114 & 0.4028 & $42.238 \%$ & $80.4825 \%$ \\
\hline NB & $78 \%$ & $22 \%$ & 0.56 & 0.2255 & 0.4655 & $45.0617 \%$ & $93.0173 \%$ \\
\hline RF & $72 \%$ & $28 \%$ & 0.44 & 0.314 & 0.4021 & $62.5339 \%$ & $80.0619 \%$ \\
\hline $\mathrm{J} 48$ & $74 \%$ & $26 \%$ & 0.48 & 0.2617 & 0.5029 & $52.2888 \%$ & $100.4931 \%$ \\
\hline
\end{tabular}

Table 3. Detailed Accuracy by class with ROI size (16x16)

\begin{tabular}{|c|c|c|c|c|c|c|c|c|c|}
\hline Classifiers & $\begin{array}{l}\text { True } \\
\text { PR }\end{array}$ & $\begin{array}{l}\text { False } \\
\text { PR }\end{array}$ & Pre & REC & F-M & $\mathrm{MCC}$ & ROC A & PRC A & CLASS \\
\hline \multirow[t]{3}{*}{ MLP } & 0.840 & 0.200 & 0.808 & 0.840 & 0.824 & 0.641 & 0.856 & 0.831 & Abnormal \\
\hline & 0.800 & 0.160 & 0.833 & 0.800 & 0.816 & 0.641 & 0.856 & 0.834 & Normal \\
\hline & 0.820 & 0.180 & 0.821 & 0.820 & 0.820 & 0.641 & 0.856 & 0.832 & $\begin{array}{l}\text { Weighted } \\
\text { Avg }\end{array}$ \\
\hline \multirow[t]{2}{*}{ NB } & 0.840 & 0.280 & 0.750 & 0.840 & 0.792 & 0.564 & 0.827 & 0.794 & Abnormal \\
\hline & 0.720 & 0.160 & 0.818 & 0.720 & 0.766 & 0.564 & 0.834 & 0.861 & Normal \\
\hline
\end{tabular}




\begin{tabular}{|c|c|c|c|c|c|c|c|c|c|}
\hline & 0.780 & 0.220 & 0.784 & 0.780 & 0.779 & 0.564 & 0.830 & 0.827 & $\begin{array}{l}\text { Weighted } \\
\text { Avg }\end{array}$ \\
\hline \multirow[t]{3}{*}{ RF } & 0.720 & 0.280 & 0.720 & 0.720 & 0.720 & 0.440 & 0.850 & 0.861 & Abnormal \\
\hline & 0.720 & 0.280 & 0.720 & 0.720 & 0.720 & 0.440 & 0.850 & 0.873 & Normal \\
\hline & 0.720 & 0.280 & 0.720 & 0.720 & 0.720 & 0.440 & 0.850 & 0.867 & $\begin{array}{l}\text { Weighted } \\
\text { Avg }\end{array}$ \\
\hline \multirow[t]{3}{*}{ J48 } & 0.760 & 0.280 & 0.731 & 0.760 & 0.745 & 0.480 & 0.758 & 0.698 & Abnormal \\
\hline & 0.720 & 0.240 & 0.750 & 0.720 & 0.735 & 0.480 & 0.758 & 0.705 & Normal \\
\hline & 0.740 & 0.260 & 0.740 & 0.740 & 0.740 & 0.480 & 0.758 & 0.701 & $\begin{array}{l}\text { Weighted } \\
\text { Avg }\end{array}$ \\
\hline
\end{tabular}

Table 4. Classifiers Summary with ROI size (20x20)

\begin{tabular}{|c|c|c|c|c|c|c|c|}
\hline Classifiers & CCI & InCCI & KS & MAE & RMSE & RAE & RRSE \\
\hline MLP & $88 \%$ & $12 \%$ & 0.76 & 0.1571 & 0.3413 & $31.3855 \%$ & $68.2018 \%$ \\
\hline NB & $86 \%$ & $14 \%$ & 0.72 & 0.1398 & 0.3736 & $27.9333 \%$ & $74.6516 \%$ \\
\hline $\mathbf{R F}$ & $84 \%$ & $16 \%$ & 0.68 & 0.1736 & 0.3034 & $34.6905 \%$ & $60.6206 \%$ \\
\hline $\mathrm{J} 48$ & $86 \%$ & $14 \%$ & 0.72 & 0.1534 & 0.371 & $30.6623 \%$ & $74.1281 \%$ \\
\hline
\end{tabular}

Table 5. Detailed Accuracy by class with ROI size (20x20)

\begin{tabular}{|c|c|c|c|c|c|c|c|c|c|}
\hline Classifiers & $\begin{array}{l}\text { True } \\
\text { PR }\end{array}$ & $\begin{array}{c}\text { False } \\
\text { PR }\end{array}$ & Pre & REC & F-M & MCC & ROC A & PRC A & CLASS \\
\hline \multirow[t]{3}{*}{ MLP } & 0.800 & 0.040 & 0.952 & 0.800 & 0.870 & 0.770 & 0.882 & 0.886 & Abnormal \\
\hline & 0.960 & 0.200 & 0.828 & 0.960 & 0.889 & 0.770 & 0.882 & 0.856 & Normal \\
\hline & 0.880 & 0.120 & 0.890 & 0.880 & 0.879 & 0.770 & 0.882 & 0.871 & $\begin{array}{l}\text { Weighted } \\
\text { Avg }\end{array}$ \\
\hline \multirow[t]{3}{*}{ NB } & 0.800 & 0.080 & 0.909 & 0.800 & 0.851 & 0.725 & 0.921 & 0.914 & Abnormal \\
\hline & 0.920 & 0.200 & 0.821 & 0.920 & 0.868 & 0.725 & 0.924 & 0.909 & Normal \\
\hline & 0.860 & 0.140 & 0.865 & 0.860 & 0.859 & 0.725 & 0.922 & 0.911 & $\begin{array}{l}\text { Weighted } \\
\text { Avg }\end{array}$ \\
\hline \multirow[t]{3}{*}{$\mathbf{R F}$} & 0.800 & 0.120 & 0.870 & 0.800 & 0.833 & 0.682 & 0.931 & 0.951 & Abnormal \\
\hline & 0.880 & 0.200 & 0.815 & 0.880 & 0.846 & 0.682 & 0.931 & 0.891 & Normal \\
\hline & 0.840 & 0.160 & 0.842 & 0.840 & 0.840 & 0.682 & 0.931 & 0.921 & Weighted \\
\hline \multirow[t]{3}{*}{ J48 } & 0.880 & 0.160 & 0.846 & 0.880 & 0.863 & 0.721 & 0.849 & 0.817 & Abnormal \\
\hline & 0.840 & 0.120 & 0.875 & 0.840 & 0.857 & 0.721 & 0.849 & 0.796 & Normal \\
\hline & 0.860 & 0.140 & 0.861 & 0.860 & 0.860 & 0.721 & 0.849 & 0.807 & $\begin{array}{l}\text { Weighted } \\
\text { Avg }\end{array}$ \\
\hline
\end{tabular}


Table 6. Classifiers Summary with ROI size (22x22)

\begin{tabular}{|c|c|c|c|c|c|c|c|}
\hline Classifiers & $\mathrm{CCI}$ & InCCI & KS & MAE & RMSE & RAE & RRSE \\
\hline MLP & $82 \%$ & $18 \%$ & 0.64 & 0.2277 & 0.4086 & $45.5008 \%$ & $81.6432 \%$ \\
\hline NB & $76 \%$ & $24 \%$ & 0.52 & 0.2497 & 0.4857 & $49.9043 \%$ & $97.0583 \%$ \\
\hline $\mathbf{R F}$ & $90 \%$ & $10 \%$ & 0.8 & 0.2314 & 0.3195 & $46.2406 \%$ & $63.8408 \%$ \\
\hline $\mathrm{J} 48$ & $88 \%$ & $12 \%$ & 0.76 & 0.1359 & 0.3409 & $27.152 \%$ & $68.1112 \%$ \\
\hline
\end{tabular}

Table 7. Detailed Accuracy by class with ROI size (22x22)

\begin{tabular}{|c|c|c|c|c|c|c|c|c|c|}
\hline Classifiers & $\begin{array}{l}\text { True } \\
\text { PR }\end{array}$ & $\begin{array}{c}\text { False } \\
\text { PR }\end{array}$ & Pre & REC & F-M & MCC & ROC A & PRC A & CLASS \\
\hline \multirow[t]{3}{*}{ MLP } & 0.720 & 0.080 & 0.900 & 0.720 & 0.800 & 0.653 & 0.779 & 0.852 & Abnormal \\
\hline & 0.920 & 0.280 & 0.767 & 0.920 & 0.836 & 0.653 & 0.779 & 0.707 & Normal \\
\hline & 0.820 & 0.180 & 0.833 & 0.820 & 0.818 & 0.653 & 0.779 & 0.779 & Weighted \\
\hline \multirow[t]{3}{*}{ NB } & 0.640 & 0.120 & 0.842 & 0.640 & 0.727 & 0.536 & 0.877 & 0.894 & Abnormal \\
\hline & 0.880 & 0.360 & 0.710 & 0.880 & 0.786 & 0.536 & 0.877 & 0.894 & Normal \\
\hline & 0.760 & 0.240 & 0.776 & 0.760 & 0.756 & 0.536 & 0.877 & 0.894 & $\begin{array}{l}\text { Weighted } \\
\text { Avg }\end{array}$ \\
\hline \multirow[t]{3}{*}{$\mathbf{R F}$} & 0.880 & 0.080 & 0.917 & 0.880 & 0.898 & 0.801 & 0.935 & 0.951 & Abnormal \\
\hline & 0.920 & 0.120 & 0.885 & 0.920 & 0.902 & 0.801 & 0.935 & 0.910 & Normal \\
\hline & 0.900 & 0.100 & 0.901 & 0.900 & 0.900 & 0.801 & 0.935 & 0.930 & $\begin{array}{l}\text { Weighted } \\
\text { Avg }\end{array}$ \\
\hline \multirow[t]{3}{*}{ J48 } & 0.800 & 0.040 & 0.952 & 0.800 & 0.870 & 0.770 & 0.849 & 0.877 & Abnormal \\
\hline & 0.960 & 0.200 & 0.828 & 0.960 & 0.889 & 0.770 & 0.849 & 0.775 & Normal \\
\hline & 0.880 & 0.120 & 0.890 & 0.880 & 0.879 & 0.770 & 0.849 & 0.826 & $\begin{array}{l}\text { Weighted } \\
\text { Avg }\end{array}$ \\
\hline
\end{tabular}

\section{Chart Title}

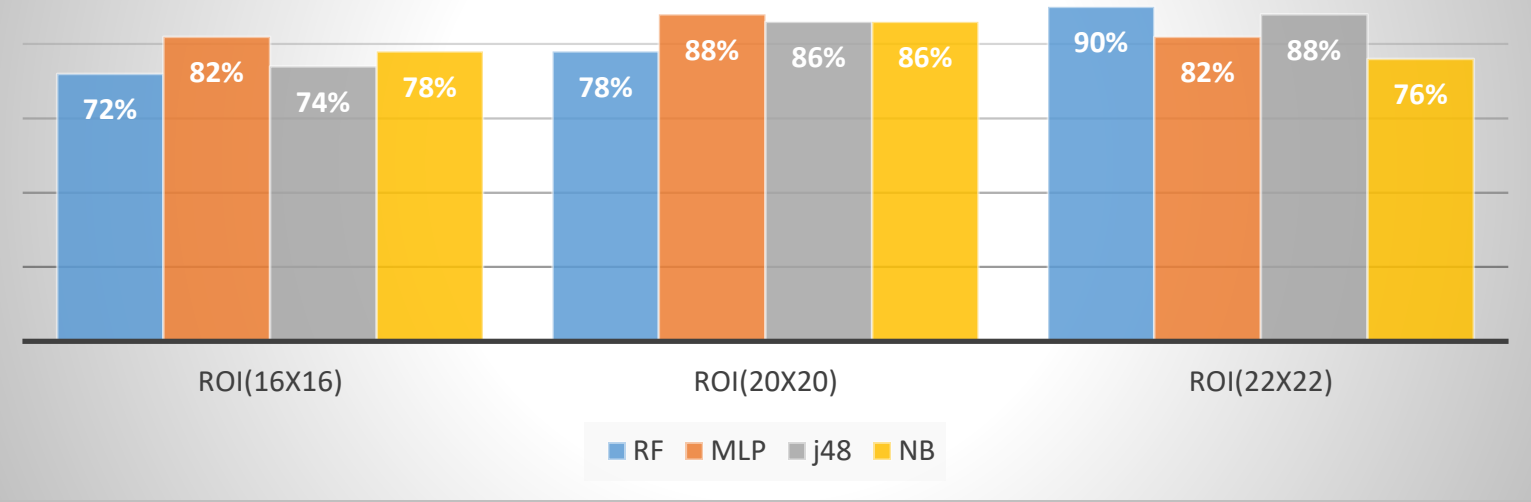

Figure 4: comparative results using four different Classifier's with ROI size (22x22) 
In Figure 4 shows the comparative analysis of three different nonoverlapping ROI sizes $16 \times 16,20 \times 20$ and $22 \times 22$ respectively with four different types of Classifier's namely Random Forest, Multi-Layer perception (MLP), j48 and Naïve Bayes are used to Classify the kidney normality's and abnormalities. The overall best performance is given Random Forest Classifier's with ROI size $22 \times 22$ to predict the $90 \%$ accuracy.

\section{CONCLUSION}

Classification of kidney stone identification using machine vision approach. In present days, researchers need to design or propose some method or system for the Classification of kidney stone identification. This research work proposed an intelligent system that Classifies kidney's normality's and abnormalities by applying ML Classifiers with the help of texture analysis, using the CT images. The goal of this research was to develop and select suitable optimized features and identify suitable Classifiers for an efficient Classification. This research explores the Classification and feature extraction technique for

\section{REFERENCES}

[1] N. Thein, . H. A. Nugroho, . T. B. Adji and . K. Hamamot, "An image preprocessing method for kidney stone segmentation in CT scan images," International Conference on Computer Engineering, Network and Intelligent Multimedia (CENIM), pp. 147-150, 2018.

[2] W. Brisbane, . M. . R. Bailey and . M. D. Sore, "An overview of kidney stone imaging techniques," Nature Reviews Urology volume 13, vol. 13, no. 2016, pp. 654-662, 2016.

[3] K. Kumar and A. , "Artificial Neural Networks for Diagnosis of," I.J. Information Technology and Computer Science, vol. 1, pp. 20-25, 2012.

[4] Y. Kazemi and S. . A. Mirroshandel, "A novel method for predicting kidney stone type using ensemble learning," Artificial intelligence in medicine, vol. 84, no. 2018, pp. 117-126, 2018.

[5] H. Zhang, C.-L. Hung, W. C.-C. Chu, P.-F. Chiu and C. Y. Tang, "Chronic Kidney Disease Survival Prediction with Artificial Neural Networks," in IEEE, Madrid, Spain, 2018.
Kidney stone identification. Results are varied due to different modalities of texture analysis that have been applied. Histogram, co-occurrence and run-length matrix features are extracted in this research. At the end of research multi-feature dataset was generated. This dataset has a combination of extracted features that are mentioned above. ML Classifier has been applied for Classification and comparative analysis of all the results (which approach is best for Classifying kidney stone identification). There are many features due to which accuracy of classification is minimized and total execution time is maximized. So, main focus has been applied on optimization process and use of an optimized feature dataset. This research also interacts with IP techniques, segmentation techniques, features extraction and reduction approach. All these steps played an imperative role in the Classification of kidney stone identification. This research concluded that more accurate and precise results can be achieved by applying ML Classifiers (Random Forest, j48, MLP and Naïve Byes) on data set generated by the fusion of multiple features (histogram co-occurrence, and runlength matrix).

[6] Ziemba, Justin B., and Brian R. Matlaga., "Epidemiology and economics of nephrolithiasis," Investigative and clinical urology, vol. 58(5), pp. 299-306, 2017.

[7] T. Rahman and M. S. Uddin, "Speckle noise reduction and segmentation of kidney regions from ultrasound image," in IEEE, Dhaka, Bangladesh, 2013.

[8] J Khan, Javed, Jun S. Wei, Markus Ringner, Lao H. Saal, Marc Ladanyi, Frank Westermann, Frank Berthold, "CLASSification and diagnostic prediction of cancers using gene expression profiling and artificial neural networCCI," Nature medicine, vol. 7(6), no. 12, pp. 673-679, 2001.

[9] Maurya, Akash, Rahul Wable, Rasika Shinde, Sebin John, Rahul Jadhav, and R. Dakshayani., "Predictive Analytics for Chronic Kidney Disease,"pp. 1-4 in IEEE, 2016.

[10] M. F. Navdeep Tangri, M. M. F. Lesley A. Stevens, P. John Griffith, M. Hocine Tighiouart, M. Ognjenka Djurdjev, M. F. David Naimark, M. F. Adeera Levin and M. Andrew S. Levey, "A predictive model for progression of chronic 
kidney disease to kidney failure," Jama, vol. 305(15), no. 11, pp. 1553-1559, 2011.

[11] Celik, Enes, Muhammet Atalay, and Adil Kondiloglu "The diagnosis and estimate of chronic kidney disease using the machine learning methods," International Journal of Intelligent Systems and Applications in Engineering, pp. 27-31, 2016.

[12] Kuo, Chin-Chi, Chun-Min Chang, Kuan-Ting Liu, Wei-Kai Lin, Hsiu-Yin Chiang, Chih-Wei Chung, Meng-Ru Ho, Pei-Ran Sun, Rong-Lin Yang, and Kuan-Ta Chen, "Automation of the kidney function prediction and CLASSification through ultrasound-based kidney imaging using deep learning," npj Digital Medicine, vol. 2(1), p. 29, 2019.

[13] Längkvist, Martin, Johan Jendeberg, Per Thunberg, Amy Loutfi, and Mats Lidén, "Computer aided detection of ureteral stones in thin slice computed tomography volumes using Convolutional Neural NetworCCI," Computers in biology and medicine, vol. 97, pp. 153-160, 2018.

[14] Abdelaziz, Ahmed, Mohamed Elhoseny, Ahmed S. Salama, and A. M. Riad, "A machine learning model for improving healthcare services on cloud computing environment," Measurement, vol. 119, no. April 2018, pp. 117128, 2018.

[15] Norouzi, Jamshid, Ali Yadollahpour, Seyed Ahmad Mirbagheri, Mitra Mahdavi Mazdeh, and Seyed Ahmad Hosseini, "Predicting renal failure progression in chronic kidney disease using integrated intelligent fuzzy expert system," Computational and Mathematical Methods in Medicine, vol. 2016, no. 2016, 2016.

[16] F. Oladeji, P. Idowu, N. Egejuru, S. Faluyi and J. Balogun, " Model For Predicting The Risk of Kidney Stone Using Data Mining Techniques," UNILAGSpace, 2019.

[17] Cui, Xiaoyu, Zeyin Zhao, Gejun Zhang, Shuo Chen, Yue Zhao, and Jiao Lu "Analysis and CLASSification of kidney stones based on
Raman spectroscopy," Biomedical optics express, vol. 9(9), pp. 4175-4183, 2018.

[18] G. R. Vásquez-Morales, S. M. MartínezMonterrubio, P. Moreno-Ger and J. A. RecioGarcía, "Explainable Prediction of Chronic Renal Disease in the Colombian Population Using Neural Networks and Case-Based Reasoning," IEEE Access, vol. 7, pp. 152900152910, 2019.

[19] F Serrat, J., Lumbreras, F., Blanco, F., Valiente, M. and López-Mesas , "myStone: A system for automatic kidney stone classification," Expert Systems with Applications, vol. 89, pp. 41-51, 2017.

[20] Murshid, Golam, Thakor Parvez, Nagani Fezal, Lakhani Azaz, and Mohammad Asif, "Data Mining Techniques to Predict Chronic Kidney Disease," International Journal of Scientific Research in Computer Science, Engineering and Information Technology, vol. 5, no. 2, pp. 2456-3307, 2019.

[21] Kamnitsas, Konstantinos, Christian Ledig, Virginia FJ Newcombe, Joanna P. Simpson, Andrew D. Kane, David K. Menon, Daniel Rueckert, and Ben Glocke, "Efficient multiscale 3D CNN with fully connected CRF for accurate brain lesion segmentation," Medical image analysis, vol. 36 , pp. 61-78, 2017.

[22] De Perrot, Thomas, Jeremy Hofmeister, Simon Burgermeister, Steve P. Martin, Gregoire Feutry, Jacques Klein, and Xavier Monte, "Differentiating kidney stones from phleboliths in unenhanced low-dose computed tomography using radiomics and machine learning," European radiology, vol. 29(9) , pp. 4776-4782, 2019.

[23] Chen, Zhaoyi, Victoria Y. Bird, Rupam Ruchi, Mark S. Segal, Jiang Bian, Saeed R. Khan, Marie-Carmelle Elie, and Mattia Prosperi, "Development of a personalized diagnostic model for kidney stone disease tailored to acute care by integrating large clinical, demographics and laboratory data: the diagnostic acute care algorithm - kidney stones (DACA-KS)," BMC 
Medical Informatics and Decision Making, vol. 18(1), p. 72, 2018.

[24] Ebrahimi, Saman, and Vladimir Y. Mariano, "Image Quality Improvement in Kidney Stone Detection on Computed Tomography Images," Journal of Image and Graphics, vol. 3(1), pp. 40-46, 2015.

[25] A. M. Hamad, "Lung cancer diagnosis by using fuzzy logic," IJCSMC,, vol. 5(3), pp. 32-41, 2016.

[26] Y. Amirgaliyev, S. Shamiluulu and A. Serek, "Analysis of Chronic Kidney Disease Dataset by Applying Machine Learning Methods," in IEEE, Almaty, Kazakhstan, 2018.

[27] Sinha, Parul, and Poonam Sinha, "Comparative Study of Chronic Kidney Disease," International Journal of Engineering Research \& Technology (IJERT), vol. 4(12), pp. 608-12, 2015.

[28] Mohammed Siyad, B., Manoj, M., Mohammed Siyad, B. and Manoj, M, "Fused features CLASSification for the effective prediction of chronic kidney disease," International Journal of Engineering \& Technology, vol. 2, pp. 44-48, 2018.

[29] M. A. M. Amanah Febrian Indriania, "SVM Optimization Based on PSO and AdaBoost to Increasing Accuracy of CKD Diagnosis," LontarKomputer:

JurnalIlmiahTeknologiInformasi, pp. 119-127, 2019.

[30] A. A. ,. P. A.Nithya, "Kidney disease detection and segmentation using artificial neural network and multi-kernel k-means clustering for ultrasound images," Measurement, vol. 149, p. 106952, 2020.

[31] P. Panwong and N. Iam-On, "Predicting transitional interval of kidney disease stages 3 to 5 using data mining method," in IEEE, Chiang Mai, Thailand, 2016.

[32] A. Martínez, D.-H. Trinh, J. E. Beze, J. Hubert, P. Eschwege, V. Estrade and L. Aguilar,
"Towards an automated classification method for ureteroscopic kidney stone images using ensemble learning," in IEEE, Montreal, QC, Canada, 2020.

[33] Black, Kristian M., Hei Law, Ali Aldoukhi, Jia Deng, and Khurshid R. Ghani., "Deep learning computer vision algorithm for detecting kidney stone composition," BJU international, 2020.

[34] Polat, Huseyin, Homay Danaei Mehr, and Aydin Cetin, "Diagnosis of chronic kidney disease based on support vector machine by feature selection methods," Journal of medical systems, vol. 41(4), p. 55, 2017.

[35] Chen, Zhaoyi, Mattia Prosperi, Vincent G. Bird, and Victoria Y. Bird, "Analysis of Factors Associated with Large Kidney Stones: Stone Composition, Comorbid Conditions, and 24-H Urine," SN Comprehensive Clinical Medicine,, vol. 1(8), pp. 597-602, 2019.

[36] Shabaniyan, Tayyebe, Hossein Parsaei, Alireza Aminsharifi, Mohammad Mehdi Movahedi, Amin Torabi Jahromi, Shima Pouyesh, and Hamid Parvin, "An artificial intelligence-based clinical decision support system for large kidney stone treatment," Australasian physical \& engineering sciences in medicine, vol. 42(3), pp. 771-779, 2019.

[37] Ma, Fuzhe, Tao Sun, Lingyun Liu, and Hongyu Jing, "Detection and diagnosis of chronic kidney disease using deep learning-based heterogeneous modified artificial neural network," Future Generation Computer Systems., vol. 111, pp. 17-26, 2020.

[38] Qadri, Salman, Dost Muhammad Khan, Farooq Ahmad, Syed Furqan Qadri, Masroor Ellahi Babar, Muhammad Shahid, Muzammil UlRehman, "A Comparative Study of Land Cover Classification by Using Multispectral and Texture Data," BioMed research international, vol. 2016, 2016. 